\title{
Implications territoriales et ethno-culturelles d'une crise volcanique : le cas de l'éruption du Mont Pinatubo aux Philippines.
}

Jean-Christophe Gaillard

Département de géographie, Université de Savoie

Résumé : Au mois de juin 1991, le Mont Pinatubo (Philippines) a connu une brusque et violente éruption. La ville de Bacolor (province de Pampanga) a été l'une des plus sévèrement touchées par les lahars qui s'écoulent chaque saison des pluies sur les piémonts du volcan. Cet article analyse les implications territoriales et identitaires de l'éruption du Mont Pinatubo pour la communauté kapampangan qui peuple cette municipalité. A la suite de cette étude, la prise en compte des facteurs ethno-culturels apparaît un élément fondamental et nécessaire de la gestion des risques et des catastrophes. Ils jouent en effet un rôle déterminant dans les réponses des populations aux crises récurrentes engendrées par les lahars et expliquent les conflits territoriaux qui ont surgi à la suite du relogement massif des victimes.

Mots clés : Mont Pinatubo, Philippines, éruption volcanique, territoire, identité, patrimoine, facteur ethno-culturel, conflit territorial.

Abstract : In June 1991, Mount Pinatubo volcano (Philippines) awakened from five-century slumber. Since then, devastating lahars, unleashed by heavy monsoon rains, have scrambled down from the flanks of the volcano. This cataclysm, viewed as one of the largest of the preceding century, wreaked huge losses for existing settlements in the vicinity. The town of Bacolor, located at the heart of the province of Pampanga, was among the most affected area. This paper aims to assess the territorial and identity implications of the Mount Pinatubo eruption for the Kapampangan ethnic group that inhabits this municipality. The responses of the populations in facing the recurrent crisis brought out by lahar onslaughts prove to be deeply influenced by the ethno-cultural factors that differentiate the Kapampangans from the other communities in the surroundings. The territorial conflicts that arose subsequently from the massive resettlement of affected populations must also be imputed to these parameters. Actually, the ethno-cultural factors turn to be a fundamental and necessary element of risk and natural disaster management.

Key words : Mount Pinatubo, Philippines, volcanic eruption, territory, identity, patrimony, ethno-cultural factor, territorial conflict.

Le Mont Pinatubo, situé sur l'île principale de Luzon aux Philippines (fig. 1), a connu, en juin 1991, une brusque et violente éruption survenue après plus de cinq siècles de sommeil. Depuis, de violents lahars (coulées de débris volcaniques), déclenchés par les pluies tropicales associées à la mousson ou au passage de typhons ainsi que par la vidange brutale de lacs, ont dévasté de nombreuses villes et villages situés sur les piémonts du volcan. Au regard des pertes matérielles colossales engendrées (sans doute supérieures à un milliard de dollars) et du nombre de personnes affectées (environ deux millions), cette catastrophe au caractère récurrent est considérée comme l'une des plus importantes du XXème siècle (Leone \& Gaillard, 1999). 
La ville de Bacolor, située au cœur de la province de Pampanga, a été parmi les secteurs les plus sévèrement touchés. En 1990, Bacolor était une municipalité de taille moyenne (67 259 habitants) située au contact entre la zone humide et rizicole du delta du fleuve Pampanga, au sud, et les terres sèches propices à la culture de la canne à sucre, au nord. Durant les trois siècles de colonisation espagnole (1571-1898), Bacolor a longtemps été la capitale de la province de Pampanga (de 1706 à 1904 exactement). Elle a même été élevée au rang de capitale des Philippines durant les deux ans d'intermède colonial britannique, entre 1762 et 1764 (Henson, 1963 ; Larkin, 1993). Les habitants de Bacolor, ou Bacoloreños, étaient très fiers de leurs illustres aînés ayant participé, à la fin du XIXème siècle, à l'émergence d'une très influente culture kapampangan (de Pampanga). Les statues à la mémoire des artistes locaux participaient à l'identité culturelle de Bacolor, baptisée symboliquement « Athènes de Pampanga ». Depuis 1861, la municipalité possédait en outre le plus ancien établissement d'enseignement professionnel d'extrême orient qui drainait des étudiants venant de l'ensemble de la province de Pampanga (Henson, 1963).

Les lahars du Mont Pinatubo ont engendré, à Bacolor, de profondes mutations territoriales et identitaires conditionnées par un certain nombre de facteurs ethno-culturels distinguant les populations kapampangans des autres habitants de la région du Mont Pinatubo. Cet article en dresse une analyse pour les quatre quartiers formant le centre historique de la ville: Cabambangan (1463 habitants), San Vicente (6373 habitants), Santa Ines (3441 habitants) et Cabetican (4866 habitants). Ce secteur, qui est le foyer de population originel de Bacolor, regroupe l'ensemble des marqueurs territoriaux de la ville. Jusqu'en 1991, ces quartiers étaient prospères et la population disposait de revenus relativement confortables par rapport à la moyenne régionale. La principale église, celle de San Guillermo, était le symbole le plus notoire de la cité. Entre 1991 et 1995, ces quatre quartiers ont été progressivement enterrés sous près de dix mètres de boue déposée par les lahars successifs de la rivière Pasig-Potrero. Ces derniers ont enseveli tout ou partie des habitations et des monuments de la ville (Crittenden \& Rodolfo, sous presse).

Dans un premier temps, on considérera les réponses des institutions et des populations aux crises liées à l'éruption et aux lahars du Mont Pinatubo. On s'attachera ensuite à montrer leurs répercussions en terme d'identité territoriale, avant d'évaluer le poids des facteurs ethnoculturels qui contribuent aussi à les forger. Nous évoquerons enfin les conflits territoriaux qui ont surgi à la suite de l'éruption.

\section{Les réponses institutionnelles à l'éruption et aux lahars du Mont Pinatubo.}

Le paroxysme de l'éruption du Mont Pinatubo (Central Luzon, Philippines), a été atteint entre les 12 et 15 juin 1991, soit deux mois après les premiers signes de réveil du volcan (fig. 1). La gestion immédiate de la crise éruptive du Mont Pinatubo a indéniablement été une réussite au regard des conséquences d'autres phénomènes d'intensité pourtant inférieure, telle l'éruption du Nevado Del Ruiz (Colombie) en 1985. Le réveil du volcan a été diagnostiqué assez tôt pour permettre la mise en place de mesures d'alerte et d'évacuation efficaces. Le nombre limité de victimes, environ 900 morts, l'atteste (près de 25000 victimes suite au principal lahar du Nevado Del Ruiz qui dévasta la ville d'Armero). L'expérience des autorités philippines en matière de gestion de crises volcaniques et la parfaite collaboration entre les scientifiques locaux et leurs homologues américains venus en renfort, ont largement contribué à ce succès reconnu au niveau mondial (Leone \& Gaillard, 1999). 
La gestion des lahars s'est avérée plus délicate et quelques événements tragiques ont jalonné la décennie. Le drame de Cabalantian (quartier de Bacolor situé à l'est du centre historique de la ville), en octobre 1995, restera le plus significatif au regard de la centaine de personnes ayant succombé à un terrible lahar de la rivière Pasig-Potrero. La récurrence des lahars et la multiplication des alertes ont été ici un facteur délicat à gérer pour les autorités qui peinaient par ailleurs à définir l'extension spatiale des phénomènes (Leone \& Gaillard, 1999).

La principale difficulté consista à réhabiliter les secteurs affectés tout en les prévenant d'autres lahars destructeurs. Deux stratégies antagonistes s'offraient aux autorités philippines : déplacer et reloger l'ensemble des populations encore menacées ou entreprendre des mesures structurelles de protection des villages exposés. Tiraillé entre les partisans des deux théories, le gouvernement de Manille opta pour une solution de compromis. Les victimes de l'éruption et des premiers lahars ont été conduites dans de vastes centres de relogement bâtis en zones sûres, alors que la plupart des chenaux d'écoulement des lahars ont été endigués.

Une structure intergouvernementale, la Task Force Mount Pinatubo, devenue, en 1992, la Mount Pinatubo Commission (MPC), fut créée afin de mettre en place la politique gouvernementale de relogement. La plupart des habitants de Bacolor, victimes des lahars de la Pasig-Potrero, furent relogés dans quatre immenses et uniformes centres de relogement (Bulaon, Madapdap, Pandacaqui et Santa Lucia), organisés autour d'une place centrale accueillant les principaux bâtiments publics (poste de police, centre de secours, terrains de jeu...) (fig. $2 \& 3$ ). Les autorités attribuèrent à chaque famille un terrain de $94 \mathrm{~m} 2$ et une petite bâtisse bétonnée équipée de sanitaires. Des kilomètres de routes et lignes électriques ainsi que plusieurs dizaines d'écoles ont été construits afin de subvenir aux besoins des victimes. Le plus important de ces centres de relogement, Madapdap, accueille plus de 7000 familles, soit très largement plus que les villages d'origine dont la population ne dépassait pas quelques milliers d'habitants. Cette politique a été accompagnée de nombreux projets visant à fournir des emplois aux personnes relogées, notamment au travers de la construction de 'mini-zones industrielles' à proximité des centres de relogement les plus importants.

Parallèlement au relogement des habitants des villages d'ores et déjà affectés, le gouvernement philippin entreprit de vastes et coûteux travaux d'endiguement de la rivière Pasig-Potrero (fig. 3). Face à l'inefficacité des premières digues, les autorités optèrent, en 1996, pour la construction d'un gigantesque projet baptisé « Megadike ». La résistance de ce système d'endiguement, toujours pas achevé au mois d'août 2001, n'a cessé d'être remise en cause, notamment par Kelvin S. Rodolfo, un scientifique américain d'origine philippine, spécialiste mondialement reconnu des lahars. De nombreuses défaillances de la digue lui ont apporté des arguments pour fustiger la politique gouvernementale. A la suite de la construction de la Megadike, la ville de Bacolor a été transformée en bassin de réception pour les lahars de la rivière Pasig-Potrero. Le système d'endiguement actuel suit en effet de très près le contour des limites communales, encloisonnant littéralement la municipalité (fig. 3). Ce choix a été largement critiqué. Fallait-il réellement sacrifier Bacolor pour protéger la capitale provinciale et régionale, San Fernando, et une autre ville de près de 100000 habitants, Guagua? Existait-il une autre solution, sachant que, quelle que soit la stratégie adoptée, Bacolor se serait toujours trouvé dans le chenal d'écoulement naturel de la rivière PasigPotrero ? De plus, dans une région où la densité de population dépasse $1500 \mathrm{hab} / \mathrm{km} 2$, il est très difficile de protéger une ville sans mettre en danger les municipalités voisines. Ce sont donc des décisions politiques, dictées par le nombre d'électeurs des différentes localités menacées, qui ont conduit à la situation actuelle dans le bassin de la rivière Pasig-Potrero 
(Rodolfo, 1995). Heureusement, les dépôts successifs des lahars ont modifié la topographie locale, surélevant ainsi le centre historique de la municipalité de Bacolor. Si bien qu'aujourd'hui les secteurs périphériques sont sans doute devenus beaucoup plus vulnérables que les alentours de l'église San Guillermo (Crittenden \& Rodolfo, sous presse).

\section{Les réponses des populations.}

Nous l'avons déjà souligné, le faible nombre de victimes de l'éruption et des lahars du Mont Pinatubo atteste aussi bien d'une gestion institutionnelle efficace des crises que du comportement coopératif des populations. Situé à près de $40 \mathrm{~km}$ du cratère, le centre historique de la municipalité de Bacolor a été épargné des plus importantes retombées aériennes de l'éruption (chutes de cendres...). . Bien préparées par une intense campagne de sensibilisation, les populations ont bien suivi les consignes des autorités précédant l'activité paroxysmale du volcan entre les 12 et 15 juin 1991 (Tayag $\&$ al, 1996). Ce ne fut pas le cas lors des crises liées aux lahars. Au fur et à mesures des saisons, de nombreux habitants de Bacolor montrèrent de plus en plus de réticences à évacuer leur domicile, effarouchés à l'idée de retourner dans des centres d'évacuation surpeuplés et foyers de nombreuses épidémies (Leone \& Gaillard, 1999).

Un petit noyau de 'rebelles' ne quitta même jamais leur domicile. Pour cela, ils développèrent des techniques originales de prévention. Ils reçurent l'appui et les conseils de K. S. Rodolfo qui leur permirent d'organiser leur lutte contre la stratégie gouvernementale d'endiguement de la Pasig-Potrero. Ils rehaussèrent leurs maisons à l'aide de pilotis en béton qui ont, jusqu'à aujourd'hui, parfaitement résisté aux différents lahars (fig. 4). Les sacs de sable, largement utilisés dans les municipalités voisines, ont été bannis car accusés d'aggraver le pouvoir destructeur des lahars. Dans une logique propitiatoire, les habitants de Bacolor se sont aussi tournés vers la religion et les prières pour protéger leur ville et leur communauté, entraînant du même coup un regain d'autorité pour l'Église catholique locale (Rodolfo, 1995; Crittenden, 2001 ; Crittenden \& Rodolfo, sous presse).

Consécutivement à l'ensevelissement de leur domicile et en dépit de leurs réticences, la plupart des habitants de Bacolor acceptèrent d'être dirigés vers les centres de relogement de Madapdap (municipalité de Mabalacat), Bulaon (San Fernando), Santa Lucia (Magalang) et Pandacaqui (Mexico). La vie à l'intérieur de ces nouvelles structures urbaines s'est rapidement avérée très difficile. Les petites maisons recouvertes de tôles sont très peu confortables, particulièrement pendant l'été philippin (avril - mai) très chaud. La difficulté pour les autorités à proposer des emplois à plusieurs milliers de personnes dans des secteurs jusqu'alors voués à l'agriculture rend le contexte économique très délicat. Avant l'éruption, $24 \%$ des victimes étaient agriculteurs. A l'intérieur des centres de relogement, celles-ci durent s'adapter et s'orienter vers de nouveaux emplois industriels et commerciaux, ou, plus souvent, vers le travail informel (petites boutiques familiales, artisanat, transports de proximité...). Plusieurs centaines d'autres Bacoloreños ont préféré s'expatrier vers les états du Golfe Persique (pour les hommes) ou vers les nouveaux pays industrialisés d'Asie du Sud-Est (pour les femmes), afin de subvenir aux besoins du reste de leur famille, restée au pays.

3 Dix ans après l'éruption : le processus de réhabilitation de Bacolor. 
Les difficultés rencontrées dans les centres de relogement conduisent aujourd'hui de nombreuses familles à retourner s'installer dans leurs villages d'origine. Ceux-ci restent parfois exposés à de possibles lahars et les conditions de vie y apparaissent très difficiles en cas de fortes pluies (Bacolor est alors transformé en un véritable cloaque). En juillet 1997, un recensement effectué par une sociologue américaine dénombrait 383 familles (soit 1755 individus) dans les quatre quartiers du centre historique de Bacolor. Depuis, ce chiffre est en très nette et continuelle augmentation. Une enquête menée en 1998, soit au tout début de la vague de retours aux villages d'origine, a montré que $60 \%$ des habitants du centre historique de Bacolor avait déjà séjourné dans un centre de relogement. 30\% d'entre eux ont cité l'absence d'emplois, puis l'éloignement au village d'origine $(21 \%)$ et l'attachement à la terre natale $(18 \%)$ comme cause première de leur retour à Bacolor (Gaillard \& al., 2001). Certaines victimes retournent dans les centres de relogement durant la saison des pluies, établissant ainsi des flux saisonniers de populations entre Bacolor et Bulaon, Madapdap, Pandacaqui ou Santa Lucia.

Ces retours aux villages d'origine sont aussi motivés par une réduction de la perception du risque. En effet, depuis, 1997, Bacolor n'a plus subi aucun lahar. Durant les quatre dernières années, les phénomènes sont restés contenus dans les chenaux d'écoulement et aucun dommage significatif n'est à déplorer, sauf pour la municipalité de Minalin, située en aval de Bacolor. Au sein des communautés restées à Bacolor ou revenues très tôt dans leurs villages d'origine, cette réduction de la perception du risque se traduit concrètement par le murage des espaces compris entre les pilotis ayant servi à surélever les maisons.

Les populations de Bacolor bénéficient aujourd'hui de programmes officiels de réhabilitation du centre historique de la municipalité. Ceux-ci étaient jusqu'alors suspendus à la délivrance d'une autorisation par l'institut philippin de volcanologie (Phivolcs). Celui-ci ayant estimé que la probabilité d'occurrence de lahars susceptibles d'affecter les alentours de l'église San Guillermo était en net recul, d'importants projets infrastructuraux ont été lancés au début de l'année 2001. Deux d'entre eux retiendront notre attention. Le premier est la construction d'une nouvelle mairie. Celle-ci a été élevée tout à côté de l'ancien bâtiment dont ne subsiste aujourd'hui que le second étage. Le nouveau maire, élu en mai 2001, a intégré l'édifice un mois plus tard. Ce geste représente un symbole très fort pour les habitants de la commune. En effet, depuis 1995, l'équipe municipale était installée dans des bâtiments préfabriqués situés dans le seul village de Bacolor (Calibutbut) épargné par les lahars, et situé en bordure d'Angeles City, à l'extrême nord est de la ville. Ce projet est en partie financé par les recettes prélevées sur l'extraction de matériel sablonneux déposé par les lahars et destiné à l'industrie du bâtiment de la région de Manille. Le retour du pouvoir municipal au centre historique de la commune devrait marquer le début d'une nouvelle vague de reflux depuis les centres de relogement. D'autant plus que la construction de la nouvelle mairie concorde avec le début des travaux de réhabilitation de l'ancienne route nationale (McArthur Highway) reliant Bacolor à San Fernando, à l'est, et Guagua, à l'ouest. Cet axe de communication était autrefois l'un des plus fréquentés de la région (en moyenne 15980 véhicules par jour en 1993), notamment par les habitants de Bacolor qui se rendaient travailler à Manille. La réhabilitation de la McArthur Highway et d'un tronçon permettant de relier le centre historique de Bacolor à la nouvelle route nationale majeure, reliant San Fernando à Olongapo City, signifie, pour de nombreux habitants des centres de relogement, un effacement de la menace liée aux lahars. Ce projet constitue, aux yeux de beaucoup, le feu vert gouvernemental à la réhabilitation de Bacolor, qui n'était jusqu'alors que l'initiative de leaders locaux. 
4 Le poids des facteurs ethno-culturels.

Outre une gestion trop technocratique de la réhabilitation à la suite des crises liées aux lahars du Mont Pinatubo (Leone \& Gaillard, 1999), le relatif échec de la politique officielle de relogement peut être imputé à la non prise en compte des facteurs ethno-culturels.

Nous avons déjà souligné que la municipalité de Bacolor est l'un des berceau de la culture kapampangan. Les Kapampangans constituent une communauté de plus de 2 millions d'individus peuplant la partie sud-ouest de la plaine centrale de Luzon, comprise entre le fleuve Pampanga, au sud, le Rio Chico de Pampanga, à l'est, et la ville de Tarlac City, au nord. Cette région comprend l'intégralité de la province de Pampanga et quelques municipalités bordières du sud de la province de Tarlac (Bamban, Concepcion, Capas et une partie de Tarlac City) et du nord de celle de Bataan (une partie de la municipalité de Dinalupihan). Les ancêtres des Kapampangans, littéralement les « habitants des berges de la rivière », seraient originaires du nord de l'île de Sumatra. Ils auraient atteint les Philippines entre -200 et -300 avant J-C., s'installant sur les berges du fleuve Pampanga qui draine la moitié sud de la plaine centrale de Luzon. Contrairement à leurs voisins Ilokanos, les Kapampangans n'ont pas manifesté la même tendance migratoire et sont longtemps restés concentrés autour du delta du fleuve Pampanga. Seule la très rentable culture de la canne à sucre, implantée par les colons espagnols, poussa certains d'entre eux, au cours des XVIIIème et XIXème siècles, à s'installer sur les terres sèches situées immédiatement au nord du delta. Si bien qu'aujourd'hui, les Kapampangans forment un isolat de population à la langue et à la culture originale, entouré par une dizaine de groupes ethno-linguistiques différents (Henson, 1963 ; Larkin, 1993).

Le très fort attachement à la terre natale manifesté par les Bacoloreños, kapampangans, doit être rapporté à leur présence séculaire sur leur territoire. La municipalité de Bacolor a été créée en 1576. Il est cependant admis que la ville était habitée bien avant l'arrivée des Espagnols (Henson, 1963). Les Kapampangans passent pour être un peuple fier et matérialiste. Le montant de leur dépenses mensuelles dépasse ainsi largement celui des autres groupes ethno-linguistiques de la région.

La difficulté d'adaptation des Bacoloreños aux centres de relogement peut donc être attribuée à ce caractère. Les Kapampangans, dont les demeures traditionnelles sont vastes et équipées d'une large cuisine, se sentent très à l'étroit dans les petites maisons construites par la MPC. Dès leur arrivée dans les centres de relogement leur premier souci a donc été de faire évoluer leur habitation, en réponse à des impératifs esthétiques autant qu'à une motivation de distinction sociale. Bulaon et Madapdap sont ainsi des chantiers permanents. Les conditions économiques difficiles qui règnent dans les centres de relogement les rebutent. De ce fait, ils imaginent plus facilement pouvoir refaire leur vie dans leur village d'origine et envisagent en masse leur retour à Bacolor.

Les facteurs ethno-culturels ont donc influencé les réponses des populations face aux crises et aux réponses institutionnelles liées aux lahars du Mont Pinatubo. Il en découle un certain nombre d'implications en terme d'identité territoriale.

5 Implications en terme d'identité territoriale.

5.1 Les populations en place. 
L'éruption du Mont Pinatubo s'est tout d'abord traduite par une pratique religieuse plus assidue. Dans le cas de Bacolor, le recours à la spiritualité traduit la manifestation d'un renforcement de l'attachement au lieu. L'un des soucis permanents des Bacoloreños fut en effet de réhabiliter les églises, saisonnièrement ensevelies sous plusieurs mètres de boue, afin de maintenir les lieux de culte, si importants dans la culture philippine où l'église sert de lien spirituel permettant de souder les communautés. Le très dynamique curé du centre historique de la ville n'a ainsi jamais cessé de prêcher dans l'église San Guillermo. Celle-ci fut pourtant recouverte par plus de six mètres de dépôts. Lorsque les conditions furent particulièrement difficiles, tel en novembre 1995, lors de la fête annuelle de la ville, une tente fut dressée devant le porche de l'église afin de permettre au prêtre d'officier (Crittenden, 2001; Crittenden \& Rodolfo, sous presse). Depuis 1995, on pénètre dans l'église par une ancienne fenêtre située au sommet du porche initial. Avec le recul de la menace due aux lahars, le prêtre a initié une campagne d'embellissement de l'édifice (dallage du sol, différentes plantations devant l'entrée...). L'église San Guillermo est aujourd'hui l'un des symboles des dommages engendrés par les lahars du Mont Pinatubo, reconnue au niveau national et érigée en ressource touristique municipale.

Parallèlement à la réhabilitation des églises, les habitants de Bacolor se sont attachés à conserver leurs autres marqueurs territoriaux, les statues et le DHVCAT ${ }^{1}$ notamment, et non les monuments ou édifices communs à l'ensemble des Philippines, tels le marché public ou les écoles. A l'image de l'église, le DHVCAT n'a cessé de fonctionner et fut transformé en centre d'évacuation au plus fort des crises liées aux lahars de la Pasig-Potrero. Les statues ont quant à elles systématiquement été exhumées afin d'apparaître aux yeux de tous.

Nous observons ainsi l'importance des symboles du culte ou des monuments qui évoquent les faits et personnages de l'histoire ou du mythe de la communauté, renforçant le rapport entre peuple et territoire (Barbina, 1993). Selon Claval (1997), « le symbole réunit ». L'éruption du Mont Pinatubo et la menace de disparition des symboles patrimoniaux ont été l'occasion, pour les Bacoloreños, d'une prise de conscience très forte de leur territoire, à l'image de ce que l'on avait pu remarquer suite au séisme du Frioul (Italie), en 1976, et, dans une moindre mesure, avec l'éruption de la soufrière de Montserrat au milieu des années 1990. Les symboles religieux ou liturgiques apparaissent ici comme des éléments essentiels pour une communauté d'habitants très croyants. Par extension, les bâtiments et la statuaire deviennent autant de signes tangibles de l'identification de la communauté aux lieux, ce qui les conduit à être considérés comme des marqueurs territoriaux essentiels. Dès lors, ils deviennent des points d'ancrage territorialisants dans la mesure où ils sont investis d'une valeur symbolique. C'est à travers la valeur religieuse et culturelle que les habitants confèrent à ces objets et au lieu, que la relation ternaire lieu / objet cultuel / société se met en place.

Quelques personnages ont joué un rôle majeur dans cette évolution : le prêtre qui fut le leader, deux conseillers municipaux et K. S. Rodolfo. Le sentiment de sacrifice occasionné par la construction de la Megadike, symbole de la politique gouvernementale aux yeux des Bacoloreños, a été un autre élément fédérateur capital dans le maintien d'une communauté particulièrement active dans le centre historique de la ville. En 1998, 50\% des habitants de Bacolor déclarait un point de vue défavorable sur le gouvernement et sa gestion des crises liées aux lahars (Gaillard \& al., 2001). Quelques graffitis inscrits sur les murs de la ville en 1995, étaient particulièrement significatifs du sentiment qui habitait quelques Bacoloreños, parmi les plus dynamiques : " par amour et attachement à la terre natale, je n'abandonnerai

\footnotetext{
${ }^{1}$ Don Honoro Ventura College of Arts and Trades
} 
pas Bacolor », " Département des Travaux Publics et des Autoroutes (DPWH), tu n'as pas de Dieu » inscrit sous le buste d'un artiste local - J.S. Soto - auteur d'une pièce intitulée «Pas de Dieu $! \gg \ldots$

\subsection{Le cas des populations relogées.}

Cet attachement très fort au passé et à la ville natale se manifeste également au sein des populations relogées à Bulaon, Madapdap, Santa Lucia ou Pandacaqui. Les habitants de Bacolor déplacés dans les centres de relogement se sont appropriés leur nouveau territoire à l'aide de marqueurs territoriaux et identitaires qui reproduisent ou rappellent la ville d'origine. A Bulaon, chaque subdivision administrative porte le nom du village ou du quartier d'où sont originaires les victimes. Les magasins et les établissements commerciaux sont baptisés en référence au lieu d'origine. Ce sont en fait tous les marqueurs territoriaux à forte valeur symbolique, notamment les églises et les écoles, mais aussi les entités administratives (les conseils de village ou de quartier), qui sont apparentés à Bacolor. La mémoire est entretenue au travers des cérémonies religieuses et des fêtes locales ou municipales de Bacolor. Chaque mois d'octobre, les Bacoloreños relogés à Madapdap, Pandacaqui, Santa Lucia et Bulaon ne manquent pas d'honorer la mémoire des victimes du terrible lahar ayant ravagé leur commune en 1995.

Mais les liens qu'entretiennent les habitants des centres de relogement avec leur ville d'origine ne se limitent pas à cette reproduction de marqueurs territoriaux. En effet, ceux-ci continuent d'entretenir d'étroites relations avec le centre historique de Bacolor, notamment à Bulaon, site de relogement le plus proche. Chaque dimanche, un certain nombre de familles se rendent à l'église San Guillermo pour assister à la messe comme elles en avaient l'habitude jusqu'en 1991. Des lignes de transports en commun directes entre les centres de relogement et San Fernando (centre de communication le plus proche de Bacolor) ont pour cela été créées. La fête municipale annuelle, 'La Naval', au mois de novembre, est un événement populaire qui continue de rassembler une grande partie des Bacoloreños, comme ce fut toujours le cas depuis 1786. La fête est un événement où le sentiment d'appartenance collective est très fort (Claval, 1997). Elle s'avère très importante à Bacolor dont la population est aujourd'hui dispersée sur plusieurs sites.

\subsection{Le rôle des principaux marqueurs territoriaux.}

Un attachement très fort au centre historique de la municipalité de Bacolor et à ses principaux repères territoriaux transparaît. Deux types de valeurs patrimoniales (Di Méo, 1998) ont ici joué le rôle de ciment communautaire au sein de la population du centre historique de Bacolor, aujourd'hui éclatée sur près d'une dizaine de sites. Tout d'abord, des réalités matérielles comme l'église San Guillermo, le DHVCAT, les statues; mais aussi des phénomènes abstraits au travers d'une histoire, d'une culture très riches et de la religion qui participent à la mémoire collective. Cette démonstration est illustrée par les liens très forts qui unissent aujourd'hui les populations déplacées à leur ville d'origine. Le cas de l'église San Guillermo est particulièrement intéressant. Ce lieu, autrefois " attribut », est aujourd'hui devenu un lieu de « condensation » au sens entendu par Debarbieux (1995). Au regard de son ancienneté et de son esthétisme, l'église San Guillermo était, jusqu'au réveil du Mont Pinatubo, un symbole matérialisant Bacolor aux yeux des habitants des communes voisines. $\mathrm{Au}$ fil des crises et des pratiques cérémonielles qui ont été l'occasion de rassemblements communautaires, ce lieu s'est chargé de sens. Le nouveau maire de Bacolor, élu au mois de mai 2001, avait bien perçu ce phénomène puisque, sur ses affiches électorales, il se présentait 
sur fond d'église San Guillermo. Celle-ci est devenu le symbole de la résistance des habitants aux dommages occasionnés par les lahars. L'église et les statues sont sans doute aussi le signe visible du passé de Bacolor, aujourd'hui révolu, mais dont on veut entretenir le souvenir au travers de leur exhumation. Ces symboles constituent ainsi des points d'ancrage à l'enracinement mémoriel du territoire, notamment pour les jeunes générations qui grandissent dans les centres de relogement. Ils permettent enfin aux Bacoloreños d'avoir une existence collective et des sites de mise en scène (Debarbieux, 1995).

Cette relation particulière au territoire n'est pas sans engendrer des conflits territoriaux. Ils se manifestent autant dans les centres de relogement que dans la ville d'origine.

\section{L'apparition de conflits territoriaux.}

\subsection{A qui appartiennent les centres de relogement?}

Le contrôle politique des centres de relogement est devenu un enjeu majeur, notamment à l'approche du transfert de compétences qui a fait suite à la dissolution de la MPC, le ler janvier 2001. L'exemple des centres de relogement de Bulaon (San Fernando) et Madapdap (Mabalacat) est particulièrement éloquent.

Bien que Bulaon soit situé sur le territoire de San Fernando, le maire de Bacolor, d'où sont originaires la plupart des victimes relogées, continue de considérer le centre de relogement comme part entière de sa juridiction. Cet aspect est doublement sensible à l'approche des élections puisque les inscriptions sur les listes électorales de l'une ou de l'autre commune conditionnent le montant d'une taxe, l'Internal Revenue Allotment (IRA), qui représente une part importante du budget municipal (le montant de l'IRA dépendant aussi de la superficie de la commune). On imagine donc bien les intérêts conflictuels pouvant exister entre le maire de la municipalité d'accueil et celui de la municipalité d'origine. Le premier fournit les services de base à la communauté en 'exil' (eau, électricité, collecte des ordures...) alors que le second justifie l'enregistrement des victimes sur ses listes électorales par la situation de catastrophe. La devise de l'ancien maire de Bacolor n'était elle pas : " on ne gouverne pas un lieu mais plutôt une communauté » ? Cette remarque permet de s'interroger sur le rôle du pouvoir dans la constitution d'un nouveau territoire. Le pouvoir politique participe en effet incontestablement, mais de façon non exclusive, à la naissance puis au développement d'un territoire (Di Méo, 1998).

A l'intérieur des centres de relogement, les conflits apparaissent de façon concrète. En période électorale, les candidats de Bacolor se succèdent à Bulaon, alors qu'une grande confusion règne à Madapdap, où se côtoient des populations de Bacolor s'étant maintenues sur les listes électorales de leur municipalité d'origine et d'autres en provenance de localités voisines votant à Mabalacat. Lors du recensement de 2000, de nombreuses victimes originaires de Bacolor ont également refusé d'être comptées au sein des municipalités d'accueil.

Ce contexte délicat reflète particulièrement bien la mentalité des victimes. D'un côté, celles-ci sont demandeuses de services à la municipalité d'accueil et comprennent difficilement que cette dernière manifeste quelque scrupule à favoriser une population qui ne leur apporte pas grand chose en termes de revenus ou de bulletins électoraux. D'un autre côté, les personnes relogées, très attachées à leur municipalité d'origine, se sentent le devoir de participer à la réhabilitation de leur ville en finançant celle-ci au travers de certaines taxes, notamment l'IRA. Leur réaction virulente face au transfert de compétence en matière de gestion des 
services de base est représentative de cet état d'esprit. La MPC a en effet remis la gestion de l'eau et de l'électricité aux municipalités d'accueil qui, logiquement, pratiquent des tarifs supérieurs afin de tenter de tirer un léger bénéfice de cette population peu encline à participer à la vie de leur commune.

\subsection{Le cas des « Laharlands ».}

Le retour des populations des centres de relogement vers leur ville d'origine devrait déclencher des conflits territoriaux dont il semble encore bien difficile d'évaluer l'ampleur. En effet, les victimes tendent à revenir sur leur parcelle d'origine, mais comment redéfinir celles-ci lorsque les villages ont été ensevelis sous parfois plus de dix mètres de sédiments ?

A l'heure actuelle, ces retours épisodiques se concentrent principalement vers le centre historique de Bacolor où subsistent les vestiges de certains marqueurs territoriaux comme l'église, le toit du marché, l'école de commerce, etc. Ces monuments permettent aux géomètres de redéfinir sans trop de problèmes les parcelles cadastrales antérieures à l'éruption. Mais qu'en sera-t-il lorsque le Phivolcs ${ }^{2}$ aura donné son accord pour la réhabilitation de l'ensemble de la municipalité de Bacolor? Au moment où des centaines de familles prendront la direction de villages où il ne subsiste aucune trace du passé, tels Duat, Balas, etc. ? La question se pose déjà dans certains villages de la municipalité voisine de Porac (Pampanga) où d'anciens propriétaires revendiquent leur parcelle à l'aide de croix et de drapeaux, ou encore grâce à des clôtures barbelées ou en bambou.

D'ici quelques années, ce phénomène sera sans doute accentué par la spéculation foncière qui ne manquera pas d'intervenir autour de la municipalité de Bacolor. En effet, la position proéminente de celle-ci est sans doute la meilleure alternative aux problèmes croissants d'inondations qui affectent les villes voisines de San Fernando et Guagua. De ce fait, de nombreux habitants de ces deux localités vont probablement chercher à acquérir des terrains situés sur la municipalité de Bacolor et surélevés par les dépôts successifs des lahars.

Afin d'anticiper ces problèmes, le gouvernement philippin a lancé une grande campagne d'enquête visant à redéfinir les propriétés et le cadastre. 50141 parcelles nécessitent d'être ainsi réétudiées. Ce projet, confié au Department of Environment and Natural Ressources (DENR), demandera beaucoup de temps et d'argent (plus de 13 millions de francs), et sera soumis aux aléas de la corruption, rampante aux Philippines.

\section{Conclusion}

L'éruption du Mont Pinatubo, et surtout les lahars qui lui ont succédé, ont engendré de profondes mutations territoriales et identitaires au sein de la communauté kapampangan. La réaction des populations de Bacolor face à la destruction de leur ville et à leur évacuation dans des centres de relogement situés sur les municipalités voisines est particulièrement significative. Les populations déplacées ont renforcé leur sentiment d'appartenance à une communauté culturelle particulière dont l'identité s'est forgée à partir d'un riche passé commun. Les quelques habitants qui se sont maintenus à proximité de l'église San Guillermo ont quant à eux joué le rôle de 'gardiens du temple'. En exhumant et réhabilitant les

\footnotetext{
${ }^{2}$ Philippine Institute of Volcanology and Seismology
} 
principaux symboles patrimoniaux, repères visibles de l'identité locale, ils ont entretenu la mémoire des Bacoloreños.

Dans le cas précis de Bacolor, le risque semble avoir soudé la communauté dispersée sur près d'une dizaine de sites différents. La menace qui a pesé pendant près de 10 ans sur la ville semble avoir aussi renforcé, ou pour le moins accentué, la valeur accordée au territoire. Ce processus a nécessité le renforcement des liens culturels et sociaux, matérialisé notamment une pratique religieuse plus assidue.

Au fil de cette étude du centre historique de la municipalité de Bacolor, on a aussi et surtout tenté de mettre en valeur le poids des facteurs ethno-culturels dans le comportement des populations face aux mesures mises en place par les autorités durant la phase de réhabilitation. Cette démonstration est encore plus significative au regard des implications territoriales de l'éruption du Mont Pinatubo pour d'autres communautés ethno-linguistiques, telles les Ilokanos ou les Aetas (Gaillard \& al., à paraître).

La prise en compte des facteurs ethno-culturels apparaît donc comme un enjeu majeur de la gestion des risques et des catastrophes d'origine naturelle. A ce jour, seules quelques rares études ont souligné que la perception des risques reflète la façon dont chacun perçoit la société (Douglas \& Wildavsky, 1983 ; Lupton, 2000), ou que les informations témoignant des dommages de catastrophes dépendent en partie du cadre culturel (D'Ercole et Pigeon, 1999). D'autres travaux ont mis en évidence des différences de comportements en terme de vulnérabilité ou face à une situation de crise, notamment aux États-Unis (Fothergill \& al., 1999), en Equateur (D’Ercole, 1996), aux Philippines (Tayag \& al., 1998 ; Insauriga, 1999), dans la Caraïbe (De Vanssay, 2001) ou même en France (Colbeau-Justin, 2001). Mais peu de chercheurs ont souligné la nécessité de les intégrer dans les mesures institutionnelles de gestion des crises. La fin du XXème siècle a pourtant vu coïncider la «décennie internationale pour la prévention des catastrophes naturelles » et la « décennie internationale des populations autochtones », toutes deux promulguées par l'Organisation des Nations Unies. Mais, malgré la concordance temporelle de ces deux manifestations internationales exceptionnelles, les peuples autochtones, et les minorités ethniques en général restent l'un des parents pauvres de la gestion des risques et des catastrophes d'origine naturelle. Alors que, comme le souligne R J-L. Breton, " aucune analyse des sociétés, aucune géographie ne peuvent se concevoir sans une analyse des cultures, de leurs structures, de leurs potentialités, de leurs évolutions, différentes de celles de leur voisines ».

Bibliographie.

Barbina G., 1993, « Les communautés ethno-linguistiques et la conscience de leur territoire », Sanguin A-L. Ed., Les minorités ethniques en Europe, Paris, L’Harmattan, p. 55-60.

Claval P., 1997, La géographie culturelle, Coll. Fac Géographie, Paris, 384p.

Colbeau-Justin L., 2001, « Désir de sécurité et attachement au territoire : le cas du risque inondation », Actes du colloque International : Risques et territoires, Atelier 3 - perceptions du risque : regards institutionnels, regards « ordinaires », Vaulx-en-Velin, 16-18/05/2001.

Crittenden K.S., 2001, "Can this town survive ? Case study of a buried Philippine town », Natural Hazards Review, 2 (2), p. 72-79. 
Crittenden K.S., Rodolfo K.S., Sous presse, «Bacolor town and Pinatubo volcano : coping with recurrent lahar disaster », Catastrophism, natural disasters, and cultural change. One world archeology series, London, Routledge.

Debarbieux B., 1995, «Le lieu, le territoire et trois figures de rhétorique », L'Espace géographique, $\mathrm{n}^{\circ} 2-1995$, p. 97-111.

D’Ercole R., 1996, «Représentation cartographique des facteurs de vulnérabilité des populations exposées à une menace volcanique : application à la région du volcan Cotopaxi (Equateur) », Bull. Inst. Fr. études andines, 25 (3), p. 479-507.

D'Ercole R., Pigeon P., 1999, «L'expertise internationale des risques dits naturels : intérêt géographique », Annales de Géographie, n 608, p. 339-357.

De Vanssay B., 2001, "Risques naturels et relations homme/environnements dans les Caraïbes », Actes du colloque International : Risques et territoires, Atelier 3 - perceptions du risque : regards institutionnels, regards « ordinaires », Vaulx-en-Velin, 16-18/05/2001, p. 7591.

Di Méo G., 1998, Géographie sociale et territoires, Coll. Fac Géographie, Paris, Nathan, $320 \mathrm{p}$.

Douglas M., Wildavsky A., 1983, Risk and culture : an essay on the selection of technical and environmental danger, Berkeley, University of California Press, 224p.

Fothergill (A.), Maestas (E.G.M.), DeRouen Darlington (J.), 1999, «Race, ethnicity and disasters in the United States : a review of the literature », Disasters, 23 (2), p. 156-173.

Gaillard J-C., D'Ercole R., Leone F., 2001, «Cartography of population vulnerability to volcanic hazards and lahars of Mount Pinatubo (Philippines) : a case study in the PasigPotrero river basin (province of Pampanga) », Géomorphologie, 3 / 2001, pp. 209-222.

Gaillard J-C., Dizon L.L., Leone F., A paraitre, "Territorial implications of the Mount Pinatubo eruption for some Aeta indigenous communities: the case of Pasig and Sacobia River basins (provinces of Pampanga and Tarlac, Philippines) », Natural Hazards Review.

Henson M.A., 1963, The province of Pampanga and its towns A.D. 1300-1963, Angeles City, M.A. Henson, 217p.

Insauriga (S.I.), 1999, Natural hazard awareness and disaster preparedness among the Bagobos of Mindanao. Master's thesis, University of Santo Tomas, Manila, 84p.

Larkin J.L., 1993, The Pampangans : colonial society in a Philippine province, Quezon City, New Day Publishers, 341p.

Leone F, Gaillard J-C., 1999, "Analysis of the institutionnal and social responses to the eruption and the lahars of Mount Pinatubo volcano from 1991 to 1998 (Central Luzon, Philippines) », Geojournal, 49 (2), p. 223-238. 
Lupton D. Ed., 2000, Risk and sociocultural theory: new directions and perspectives, Cambridge, Cambridge University Press, 191p.

Rodolfo K.S., 1995, Pinatubo and the politics of lahars, Quezon City, University of the Philippines Press, 341p.

Tayag J.C., Insauriga S.I., Ringor A., Belo M., 1996, «People's response to eruption warning : the Pinatubo experience, 1991-1992 », in Newhall C.G., Punongbayan R.S., Eds., Fire and mud: eruption and lahars of Mount Pinatubo, Philippines, Seattle, University of Washington Press, p. 87-106.

Tayag J.C., Coruna D., Dela Cruz J., 1998, Natural disaster management among the negritos of Luzon, Quezon City, Phivolcs, 80p.

Université de Savoie,

Département de géographie,

Campus scientifique,

73376 Le Bourget-du-Lac Cedex

jean-christophe.gaillard@univ-savoie.fr 